\title{
Evaluation of Wall Boundary Conditions For Impedance Eduction Using a Dual-Source Method
}

\author{
W. R. Watson* and M. G. Jones ${ }^{\dagger}$ \\ NASA Langley Research Center, Hampton, Virginia 23681-2199
}

\begin{abstract}
The accuracy of the Ingard-Myers boundary condition and a recently proposed modified Ingard-Myers boundary condition is evaluated for use in impedance eduction under the assumption of uniform mean flow. The evaluation is performed at three centerline Mach numbers, using data acquired in a grazing flow impedance tube, using both upstream and downstream propagating sound sources, and on a database of test liners for which the expected behavior of the impedance spectra is known. The test liners are a hard-wall insert consisting of $12.6 \mathrm{~mm}$ thick aluminum, a linear liner without a facesheet consisting of a number of small diameter but long cylindrical channels embedded in a ceramic material, and two conventional nonlinear liners consisting of a perforated facesheet bonded to a honeycomb core. The study is restricted to a frequency range for which only plane waves are cut on in the hard-wall sections of the flow impedance tube. The metrics used to evaluate each boundary condition are 1) how well it educes the same impedance for upstream and downstream propagating sources, and 2) how well it predicts the expected behavior of the impedance spectra over the Mach number range. The primary conclusions of the study are that the same impedance is educed for upstream and downstream propagating sources except at the highest Mach number, that an effective impedance based on both the upstream and downstream measurements is more accurate than an impedance based on the upstream or downstream data alone, and that the Ingard-Myers boundary condition with an effective impedance produces results similar to that achieved with the modified Ingard-Myers boundary condition.
\end{abstract}

\section{Nomenclature}

$[A],\{X\},\{G\}=$ global stiffness matrices, vector of unknown parameters, and vector containing source effects

$B, F \quad=$ momentum transfer coefficient due to mean flow $\left(B_{R}+i B_{I}\right)$ and objective function

$c_{0}, M_{0}, \rho_{0}=$ speed of sound, uniform flow Mach number, and mean static density

$f, \omega, k=$ excitation frequency, angular frequency, and freespace wavenumber

$H, L, W \quad=$ height, length, and width of computational domain

$i, p(z, x) \quad=$ unit imaginary number $(\sqrt{-1})$ and $2 \mathrm{D}$ acoustic pressure field in duct

$L_{1}, L_{2} \quad=$ locations of leading and trailing edge of test liner

nwall, $z_{J} \quad=$ number of lower wall microphones and axial location of a lower wall microphone

$x, y, z=$ vertical coordinate, horizontal coordinate, and axial coordinate

$\| \quad=$ modulus of a complex quantity

$\beta, \zeta=$ normalized admittance and normalized impedance of test liner

$\theta, \chi \quad=$ normalized resistance and normalized reactance of test liner $(\zeta=\theta+i \chi)$

$\sigma, \kappa=$ normalized conductance and normalized susceptance of test liner $(\beta=\sigma+i \kappa)$

Subscripts:

$D, U=$ a downstream source and an upstream source quantity

$e, s \quad=$ an exit plane and a source plane quantity

$F E M$, Meas $=$ a finite element computed and a measured quantity

$J \quad=$ lower wall microphone counter $(J=1,2,3 \ldots$ nwall $)$

\footnotetext{
${ }^{*}$ Senior Research Scientist, Research Directorate, Computational AeroSciences Branch, Liner Physics Group, Associate Fellow AIAA.

${ }^{\dagger}$ Senior Research Scientist, Research Directorate, Structural Acoustics Branch, Liner Physics Group, Associate Fellow AIAA.
} 


\section{Introduction}

It is often convenient to use an inviscid mean flow model that allows for slip at the wall of a flow duct when modeling sound propagation. In this case, a vanishing thin vortex sheet adjacent to the wall is used to separate the uniform mean flow in the main core from the zero grazing flow at the surface of the material. For the last half century there has been considerable discussion over the form of the impedance boundary condition for mean flow models with slip. Until the late 1950's, continuity of the normal component of acoustic particle velocity across the vortex sheet was the preferred boundary condition. ${ }^{1}$ However, in 1959 Ingard $^{2}$ derived a boundary condition that used continuity of the normal component of acoustic particle displacement across the vortex sheet. Ingard's boundary condition was restricted to a planar boundary in a uniform axial mean flow field. Myers ${ }^{3}$ extended the Ingard boundary condition to non-uniform mean flows and wall treatments with curvature. Therefore, for a period of time, two boundary conditions (continuity of acoustic particle velocity and continuity of acoustic particle displacement) were available to model the effects of an impedance boundary when the mean flow model contained slip. Because these boundary conditions led to different solutions, the question naturally arose as to which provides the best approximation to experimental data. As is detailed in the paper by Nayfeh et al., ${ }^{4}$ a number of investigators compared results of both boundary conditions to experimental data. The results were mixed, with some investigators indicating that for certain mean flows and frequency regimes one boundary condition is better than the other.

From the beginning of the 1980's until the early part of the 21st century, some of the most well-known noncommercial $^{5,6}$ and commercial ${ }^{\text {a }}$ duct acoustic codes adopted the boundary condition that was developed by Myers ${ }^{3}$ for use with their codes. The reason for this decision appears to be based on the work of Eversman and Beckemeyer ${ }^{7}$ in which it was demonstrated that in the limiting case as the boundary layer thickness tends to zero, the boundary condition reduces to that of continuity of particle displacement. Thus, for at least a brief period in history, the question of the proper wall impedance boundary condition to be used for a mean flow model with slip seems to have been resolved. Beginning with the early part of the 21 st century, two challenges arose concerning the efficacy of the Ingard ${ }^{2}$ and the Myers ${ }^{3}$ boundary conditions. These two challenges are described below.

The first challenge to the Ingard-Myers ${ }^{\mathrm{b}}$ boundary condition has to do with the possibility of instabilities (i.e., modes that grow exponentially as they propagate along the axis) that were reported by several researchers (see for example Ref. 8). These findings presented a dilemma for liner designers because these instabilities have not been observed with the conventional liners installed in commercial aircraft engines, and have rarely (see for example Ref. 9) been observed in laboratory experiments. This dilemma seems to have been resolved in a recent paper by Rienstra and Darau, ${ }^{10}$ in which they showed that the instabilities have not been seen in commercial aircraft applications because the boundary layer thicknesses in commercial aircraft engines were considerably larger than required to excite the instabilities. Furthermore, the paper indicated there was a precise combination of core Mach number, mean boundary layer thickness, excitation frequency, and source modal content that was required to excite these instabilities in laboratory experiments. This could explain why the instabilities are rarely seen in laboratory experiments.

The second challenge to the Ingard-Myers boundary condition was presented in a recent experimental paper by Renou and Auregan. ${ }^{11}$ In their paper, Renou and Auregan used Prony's method ${ }^{12}$ to educe the impedance of a conventional liner using the Ingard-Myers boundary condition under the assumptions of uniform flow. They educed different impedances for an upstream and downstream source, although the incident sound pressure level and the mean flow Mach number were held constant for both sources. Because the liner impedance should not be dependent on whether the source is upstream or downstream, ${ }^{11}$ it was concluded that "the Ingard-Myers boundary condition fails to predict with accuracy the acoustic behavior in a lined duct with flow." They presented results for a conventional liner achieved at a single flow speed to support this conclusion. Renou and Auregan then proposed a modified Ingard-Myers boundary condition that was considered to be more accurate.

The purpose of this paper is to perform a more exhausive evaluation of the modified Ingard-Myers boundary condition proposed by Renou and Auregan ${ }^{11}$ using both upstream and downstream sources under the assumption of uniform mean flow. A similar evaluation of the Ingard-Myers boundary condition is also performed. To this end, four test liners (for which the expected behavior of the impedance spectra is known) are inserted into the NASA Langley Research Center (LaRC) Grazing Flow Impedance Tube (GFIT). The impedance spectra of each test liner is then educed for an upstream and downstream source. The study is conducted at three centerline Mach numbers (Mach 0.0, 0.3 , and 0.5 ) and for excitation frequencies at which only planes waves are cut on in the hard-wall sections of the GFIT. As usual, the cut-on modes are defined as those modes that are not attenuated as they propagate along a hardwall section of the duct. The metrics used to evaluate the accuracy of the boundary condition are how well it predicts

\footnotetext{
${ }^{a}$ Actran Aeroacoustics, http://www.fft.be/index.php?pageID=4\&itemID=17 [cited April 2012]

${ }^{\mathrm{b}}$ The boundary condition derived by Myers reduces to that of Ingard for uniform flow over a plane boundary. It has become customary, therefore, to refer to the boundary condition presented by $\mathrm{Myers}^{3}$ as the Ingard-Myers boundary condition. This notation is followed in this study.
} 
the the same impedance for an upstream and downstream sound source, and how accurately it predicts the expected behavior of the impedance spectra (for each test liner) over the Mach number range. Impedances are educed using the objective function method of impedance eduction. This impedance eduction method has been well documented for an upstream sound source over the last decade ${ }^{13-17}$ and has been validated by other impedance eduction methods that employ mode synthesis. ${ }^{12,18}$ However, in the current study, modifications are proposed to this methodology that accommodate both upstream and downstream sources.

This paper is organized into seven sections. Section II introduces the governing differential equations and boundary conditions that are solved to educe the impedance of the test liners. Section III introduces the new features of the impedance eduction technique. These include the introduction of a set of constraints that allows an effective impedance to be educed that simultaneously satisfies both the upstream and downstream source measurements and the use of a global search process that is based upon the genetic algorithm. Section IV describes the flow impedance tube along with the measurements that are required to evaluate each boundary condition. Section V describes the database of test liners that are used to test the efficacy of the boundary condition. Results are presented in section VI and the primary conclusions of the paper are presented in section VII.

\section{Governing Differential Equation and Boundary Conditions}

The measured data needed to perform the current study are acquired in the LaRC GFIT. A full description of the GFIT is given in a previous paper. ${ }^{19}$ A sketch of the LaRC GFIT and its instrumentation is given in Fig. 1 and the rectangular flow duct (computational domain within the GFIT for which the analysis is performed) is illustrated in Fig. 2 for an upstream source. The portion of the upper wall between $z=L_{1}$ and $z=L_{2}$ contains the test liner and the remainder of the duct consists of rigid walls. The test liner has an unknown, but uniform, normalized impedance, $\zeta$, that is normalized with the characteristic impedance, $\rho_{0} c_{0}$, of the air flowing in the duct. Here, $c_{0}$ is the sound speed and $\rho_{0}$ is the mean static density of the air flowing in the duct. It is also assumed that the mean flow speed is uniform and is directed from left to right along the axis of the duct. Note that the source and termination planes of the computational domain for the upstream source configuration are located at $z=0$ and $z=L$, respectively. The downstream source configuration is achieved by relocating the sound source to $z=L$ so that the sound propagates against the flow and then exits the computational domain at $z=0$. Both the upstream and downstream source are assumed composed of a single tone at a frequency such that no higher-order modes propagate in the spanwise direction (i.e., the $y$ direction) in the hard-wall sections, thereby allowing the analysis to be restricted to the $(z, x)$-plane.

The acoustic pressure field, $p$, propagating through the flow duct (see Fig. 2) under the assumed conditions satisfies the convected Helmholtz equation

$$
\left(1-M_{0}^{2}\right) \frac{\partial^{2} p(z, x)}{\partial z^{2}}+\frac{\partial^{2} p(z, x)}{\partial x^{2}}-2 i k M_{0} \frac{\partial p(z, x)}{\partial z}+k^{2} p(z, x)=0
$$

where $k=\omega / c_{0}$ is the freespace wavenumber, $M_{0}$ is the uniform flow Mach number, and a time dependence of the form $e^{i \omega t}$ is assumed. As usual, the angular frequency, $\omega$, and excitation frequency, $f$, are related in the usual manner (i.e., $\omega=2 \pi f$ ). The convected Helmholtz equation can be solved to obtain the acoustic pressure field once the source, termination plane, and wall impedance boundary conditions are prescribed.

The boundary condition at the rigid lower wall is that the normal component of the acoustic pressure gradient must vanish

$$
\frac{\partial p(z, 0)}{\partial x}=0
$$

A source plane microphone located upstream of the liner at $z=0$ is flush-mounted in the hard-wall section opposite the test liner. This source plane microphone is used to measure the acoustic pressure profile, $p_{s}(x)$. This acoustic pressure profile is then used as the source plane boundary condition

$$
p(0, x)=p_{s}(x)
$$

Because the excitation frequency is kept below the cut on of higher order modes in the hard-wall sections, the acoustic pressure at the source plane microphone, $p(0,0)$, provides a measurement of the sound source pressure profile (i.e., $\left.p_{s}(x)=p(0,0)\right)$. Similarly, there is a lower wall microphone that is flush mounted in the lower wall at the termination plane. The acoustic pressure at this termination plane microphone $p(L, 0)$, provides a measurement of the termination plane acoustic pressure profile (i.e., $p_{e}(x)=p(L, 0)$ ) that is used as the termination plane boundary condition

$$
p(L, x)=p_{e}(x)
$$


The modified Ingard-Myers wall impedance boundary condition for the locally-reacting liner has been recently derived by Renou and Auregan ${ }^{11}$ and is implemented with the impedance eduction technique used in this study. For the upper wall, the modified Ingard-Myers boundary condition is

$$
-\frac{\partial p(z, H)}{\partial x}=i k\left[\frac{p(z, H)}{\zeta}\right]+(2-B) M_{0} \frac{\partial}{\partial z}\left[\frac{p(z, H)}{\zeta}\right]+\frac{(1-B) M_{0}^{2}}{i k} \frac{\partial^{2}}{\partial z^{2}}\left[\frac{p(z, H)}{\zeta}\right]
$$

where, $\zeta=\theta+i \chi$, is the unknown normalized impedance of the test liner. Here, $\theta$ is the normalized resistance and $\chi$ is the normalized reactance of the liner. The normalized admittance, $\beta=1 / \zeta$, is taken as zero for the rigid wall sections of the upper wall.

Five important observations are worth noting concerning the modified Ingard-Myers boundary condition given in Eq. (5): 1) the complex constant, $B=B_{R}+i B_{I}$, introduces two additional parameters $\left(B_{R}\right.$ and $\left.B_{I}\right)$ that will be educed, 2) these two additional parameters account for the transfer of momentum by molecular and turbulent viscosities ${ }^{11}$ into the lined wall by the mean flow, 3 ) when $B=0$, the boundary condition reduces to the continuity of the normal component of acoustic particle displacement ${ }^{1}$ across a thin vortex sheet near the lined wall and is identical to the Ingard-Myers boundary condition, 4 ) when $B=1$, the boundary condition reduces to the continuity of the normal component of acoustic particle velocity ${ }^{1}$ across the vortex sheet, and 5) when the flow is turned off $\left(M_{0}=0\right)$ or the wall is rigid $(\beta=0)$, no momentum is transferred into the liner by the mean flow so that $B$ need not be specified.

\section{The Impedance Eduction Method}

The objective function method of impedance eduction as implemented in this paper solves the boundary value problem defined by Eqs. (1)-(5) for both an upstream and a downstream sound source to educe the impedance, $\zeta$, and the mean flow transfer coefficient, $B$. Each problem is solved numerically using the finite element method (FEM). The impedance boundary condition is incorporated in the FEM analysis in weak form and cubic Hermite polynomials are used as the basis functions. First, the upstream source is switched on (with the downstream source inactive) and the source plane acoustic pressure, the termination plane acoustic pressure, and the lower wall acoustic pressure data are measured. The finite element solution for the upstream source is then obtained from the solution to the finite element matrix equation

$$
\left[A\left(\zeta_{U}, B_{U}\right)\right]\left\{X_{U}\right\}=\left\{G_{U}\right\}
$$

where the subscript, $U$, denotes the upstream source, $\left\{X_{U}\right\}$ is a vector containing the acoustic pressure solution at the finite element nodes, $\left\{G_{U}\right\}$ is a vector containing the source and termination plane acoustic pressures, and $\left[A\left(\zeta_{U}, B_{U}\right)\right]$ is the coefficient matrix for the finite element analysis. Next, the downstream source is switched on (with the upstream source inactive) and the source plane acoustic pressure, the termination plane acoustic pressure, and the lower wall acoustic pressure data are measured. The finite element solution for the downstream source is then obtained from the solution to the finite element matrix equation

$$
\left[A\left(\zeta_{D}, B_{D}\right)\right]\left\{X_{D}\right\}=\left\{G_{D}\right\}
$$

where the subscript, $D$, denotes the downstream source solution.

The FEM solutions for the upstream and downstream sound sources are now constrained to give the same liner impedance, $\zeta$, and mean flow momentum transfer coefficient, $B$,

$$
[A(\zeta, B)]\left\{X_{U}\right\}=\left\{G_{U}\right\},[A(\zeta, B)]\left\{X_{D}\right\}=\left\{G_{D}\right\}
$$

Note that once the solution to each matrix equation is constrained to give identical impedances and mean flow momentum transfer coefficients (Eq. (8)), the coefficient matrix for each problem is identical. This means that the coefficient matrix, $[A(\zeta, B)]$, needs to be inverted only once so that the solution to the downstream source problem is obtained for only a small fraction of that required to solve the upstream source problem (it is assumed that the upstream source problem is solved first).

The unknown normalized impedance, $\zeta$, and mean flow momentum transfer coefficient, $B$, satisfying Eq. (8) are now educed by an iterative process. This iterative process is constructed so that the educed values of the liner impedance, $\zeta$, and mean flow momentum transfer coefficient, $B$, reproduce the acoustic pressure field measured on the lower wall for both sound sources. This is achieved by minimizing the dual-objective function that contains four design variables

$$
F\left(\theta, \chi, B_{R}, B_{I}\right)=F_{U}\left(\theta, \chi, B_{R}, B_{I}\right)+F_{D}\left(\theta, \chi, B_{R}, B_{I}\right)
$$


The upstream and downstream objective functions are defined as the L2-norms of the difference between the measured and the acoustic pressure predicted by FEM at the microphone locations, $z=z_{J}$

$$
\begin{aligned}
& F_{U}\left(\theta, \chi, B_{R}, B_{I}\right)=\sqrt{\sum_{J=1}^{n w a l l}\left\|\left.p_{U}\left(z_{J}, 0\right)\right|_{\text {Meas }}-\left.p_{U}\left(z_{J}, 0\right)\right|_{\text {FEM }}\right\|^{2}} \\
& F_{D}\left(\theta, \chi, B_{R}, B_{I}\right)=\sqrt{\sum_{J=1}^{n w a l l}\left\|\left.p_{D}\left(z_{J}, 0\right)\right|_{\text {Meas }}-\left.p_{D}\left(z_{J}, 0\right)\right|_{\text {FEM }}\right\|^{2}}
\end{aligned}
$$

where nwall is the number of microphones that are flush mounted into the lower wall, $\left.p\left(z_{J}, 0\right)\right|_{\text {Meas }}$ is the measured acoustic pressure for the microphone located at $z=z_{J},\left.p\left(z_{J}, 0\right)\right|_{\text {FEM }}$ is the acoustic pressure predicted at the microphone using the FEM, and \|\| denotes the modulus of a complex quantity. In the objective function method of impedance eduction, the normalized resistance and reactance of the liner are selected such that the objective function $F\left(\theta, \chi, B_{R}, B_{I}\right)$ is minimized. Note that in our previous works, ${ }^{13-17}$ the impedance was educed using data for the upstream source only. This was done because data for the downstream source was not available. However, in the current effort, the effects of data for the upstream and downstream sources are considered.

The primary complication introduced by the four design variables $\left(\theta, \chi, B_{R}, B_{I}\right)$ used in the current study is that a local optima $(\theta, \chi)$ exists for each combination of $B_{R}$ and $B_{I}$. This precludes the use of a local, gradient-based optimizer such as Stewart's adaptation of the Davidon-Fletcher-Powell (SDFP) optimization algorithm, ${ }^{20}$ which is the primary optimizer used in previous studies. To overcome this difficulty, we use a genetic algorithm (GA) as the optimizer. ${ }^{21}$ The GA is an evolutionary combinatorial search technique whose evolutionary process is based on survival of the fittest. In contrast to the SDFP optimization algorithm, the GA does not require calculation of the gradient of the objective function, nor does it require an initial starting location. Furthermore, the GA obtains the global optimum. The principal drawback to the GA is that it requires significantly more function evaluations than the SDFP. To overcome this difficulty, the GA is run in parallel (i.e., several function evaluations are conducted simultaneously) to reduce wall clock time. A full description of the GA is beyond the scope of this paper; however, other salient features that are incorporated here that are discussed in more detail in Ref. 22 include, 1) a population size of fifty acoustic liners, 2) an evolutionary process using tournament-style selection and uniform crossover, 3) creep and jump mutations, and 4) an elitist strategy.

\section{Measured Data}

Measured data for this study are acquired in the GFIT. The GFIT has a cross-sectional geometry of $51 \mathrm{~mm}$ by $64 \mathrm{~mm}\left(W=51 \mathrm{~mm}, H=64 \mathrm{~mm}\right.$ ), the leading and trailing edges of the liner are located at $L_{1}=0.305 \mathrm{~m}$ and $L_{2}=0.711 \mathrm{~m}$, respectively, and the computational domain is $1.016 \mathrm{~m}$ long (i.e., $z=L=1.016 \mathrm{~m}$ ). Lower wall acoustic pressure data needed to educe the impedance are acquired at 51 ( $n$ wall $=51$ ) axial locations along the lower wall. Each lower wall microphone is separated by a distance of either 0.0127 or $0.0254 \mathrm{~m}$ from its nearest neighbor. The GFIT is configured with 18 acoustic drivers. These drivers are mounted in pairs, with two drivers mounted in opposite walls, and are generally positioned upstream of the test section. For the current test, 6 acoustic drivers ( 3 pairs) were moved downstream of the test section. These drivers were demonstrated to be capable of providing all of the sound level needed for the current tests and are tuned so that the source pressure has an incident sound pressure level of $130 \mathrm{~dB}$ at the respective source planes ( $z=0$ for the upstream source and $z=L$ for the downstream source). The FEM analysis program uses a spatial grid of eighty evenly spaced cubic elements in the axial direction (the $z$ direction) and twentyfive evenly space elements in the transverse direction (the $x$ direction) to resolve the acoustic field. Grid refinement studies indicate that further refinement of the spatial grid produces insignificant changes in educed impedance for plane wave sources. Mean flow profiles with centerline values of $0.0,0.3$, and 0.5 are acquired at axial planes just upstream $\left(z=L_{1}\right)$ and downstream $\left(z=L_{2}\right)$ of the test liner. The average values of the upstream and downstream profiles are obtained by integrating each profile across the upstream and downstream planes. Linear interpolation is used to determined the average uniform-flow Mach numbers at $z=\left(L_{1}+L_{2}\right) / 2$. These uniform flow Mach numbers are used as the values of $M_{0}$ for results reported in this study. 


\section{Database of Test Liners}

A database consisting of four test liners (one hard test liner and three soft test liners) was chosen for use in the current investigation. Each test liner is chosen to test the efficacy of the Ingard-Myers and modified Ingard-Myers boundary conditions. The first test liner is a hard-wall insert consisting of a $12.7 \mathrm{~mm}$ thick aluminum plate. The hard-wall insert provides a known admittance. The hard-wall insert results are presented in terms of the normalized admittance, $\beta=1 / \zeta$, because the resistance and reactance values are infinite in value. Here, the admittance is decomposed into its real and imaginary parts, $\beta=\sigma+i \kappa$, where $\sigma$ and $\kappa$ are the normalized conductance and susceptance, respectively. These parameters are expected to be nearly zero for our $12.7 \mathrm{~mm}$ thick sample of aluminum. A schematic of the soft test liners is given in Fig. 3 and each is described in detail in the following two paragraphs.

The second test liner is a ceramic tubular liner (see Fig. 3a) that has been studied extensively over the last 15 years. This liner is chosen because its impedance has been demonstrated to be nearly linear with respect to incident sound pressure level (SPL) and is nearly independent of the grazing flow Mach number. The ceramic tubular liner consists of parallel, cylindrical channels embedded in a ceramic matrix. These channels, with diameters of $0.6 \mathrm{~mm}$, are perpendicular to the exposed surface and provide a surface porosity of $57 \%$. The $85.6 \mathrm{~mm}$ deep channels are rigidly terminated such that each is isolated from its neighbor to ensure a locally-reacting structure. The channel diameter is small enough that grazing flow effects are insignificant relative to internal viscous losses. This liner also provides an impedance spectrum that varies over a range typically observed in aircraft engine nacelle liners.

The third and fourth test liners are conventional, perforate-over-honeycomb liners as illustrated in Fig. 3b. The mechanical structure of a conventional liner consists of a single-layer, perforate-over-honeycomb liner geometry that is representative of liners commonly used in current aircraft engine nacelles. The third liner has a facesheet with $0.991 \mathrm{~mm}$ diameter holes and a thickness of $0.635 \mathrm{~mm}$, which is bonded to a $38.18 \mathrm{~mm}$ deep cavity consisting of a $9.5 \mathrm{~mm}$ diameter hexcel honeycomb core, and is terminated with a rigid back plate. The surface porosity is $8.7 \%$. The fourth test liner is nearly identical to the third, but has a surface porosity of $13.2 \%$. These liners are designed so that the zero flow (i.e., $M_{0}=0$ ) resistance should be small compared to that of the ceramic tubular liner. These liners are also chosen because their impedances are known to be nonlinear with respect to the incident SPL and are also dependent upon the mean flow Mach number. That is, at a fixed incident SPL (as used here), an increase of the mean flow Mach number, $M_{0}$, is expected to increase the resistance of these liners. Finally, it is noted that although both conventional liners were analyzed, the conclusions for both liners are similar. Therefore in the result section of this paper, results are presented only for the low surface porosity conventional liner (i.e., the third liner).

\section{Results and Discussion}

In this section, the Ingard-Myers ${ }^{3}$ wall impedance boundary condition is evaluated on each test liner using measured data obtained for 1) the upstream source, 2) the downstream source, and 3) both sources. For convenience, this last option will be referred to as the 'dual-source' in the succeeding discussion. The dual-source objective function is minimized when both the upstream and downstream source data are used together. Readers are reminded that when both the upstream and downstream source data are used together (dual-source), an effective impedance is educed. Following the evaluation of the Ingard-Myers boundary condition, a similar evaluation is performed on the modified Ingard-Myers boundary condition. To educe the impedances using the Ingard-Myers boundary condition, the genetic algorithm was terminated after 100 generations. Because a population of fifty liners was used at each generation, this means that 5,000 function evaluations were used. However, the genetic algorithm data was examined at each generation, and it was found to have converged after somewhere between twenty and forty generations (depending on the Mach number and frequency). Modified Ingard-Myers boundary condition results were terminated after 200 generations, using the same number of liners in the population as was used in the Ingard-Myers boundary condition study (i.e., fifty liners). Thus 10,000 function evaluations were used to educe the impedance for the modified Ingard-Myers boundary condition using the genetic algorithm. After reviewing the genetic algorithm data at each of the 200 generations, the algorithm was observed to converge with the modified Ingard-Myers boundary condition between eighty and one hundred generations (again, depending on the Mach number and frequency). The increase in the number of generations needed to converge for the modified Ingard-Myers boundary condition (when compared to that required for the Ingard-Myers) was expected because the modified Ingard-Myers boundary condition contains more design variables. 


\section{A. The Ingard-Myers Boundary Condition}

\section{Hard-wall Insert}

Figure 4 shows the educed normalized conductance, $\sigma$, and susceptance, $\kappa$, for the flow-off condition $\left(M_{0}=0.0\right)$ with the hard-wall insert installed. The normalized conductance and susceptance values educed with each source are nearly identical. When the dual-source data is used to educe an effective admittance (the curve containing the open circles), no benefit is observed. Generally speaking, excellent comparison between the educed normalized conductance and susceptance and the exact hard-wall values $(\sigma=\kappa=0.0)$ are obtained in each case. A small nonzero value of normalized conductance and susceptance is observed at the frequencies greater than $1.5 \mathrm{kHz}$, but this could be due to the fact that the aluminum plate is not perfectly rigid. The good agreement between the educed normalized conductance and susceptance and the exact values gives further credence to the objective function method of impedance eduction. It is of interest to point out that over the frequency range of interest and for the flow-off condition, no higher-order modes are cut on (i.e. propagate without decay) in the hard-wall sections of the GFIT.

Figure 5 shows results for the hard-wall insert at Mach $0.3\left(M_{0}=0.3\right)$. There is also little discrepancy between the normalized conductance and susceptance educed using the upstream and downstream sources, except at the high frequency end $(f \geq 2.5 \mathrm{kHz})$ of the spectrum for the upstream source. It is noted that at this Mach number and frequency, a higher-order mode is cut on in the hard-wall sections of the GFIT for the upstream source. Because only plane waves are assumed cut on in the impedance eduction model, this could accounts for the discrepancy at the high frequency end of the spectrum for the upstream source. Note also that for this flow condition $\left(M_{0}=0.3\right)$, the scatter that occurs for frequencies of $2.5 \mathrm{kHz}$ and above for the upstream source does not occur with the effective impedance educed with the dual-source. At this Mach number, very good agreement between the educed conductance and susceptance and the exact value of zero is obtained except at the high frequency end of the spectrum where higher order modes that are not accounted for in the impedance eduction model are cut on.

Figure 6 shows results for the hard-wall insert at the highest Mach number $\left(M_{0}=0.5\right)$. At this Mach number, there is significant scatter between the normalized susceptance educed for the upstream and downstream source (see Fig. 6). Note also that when the dual-source data are used to educe effective conductance and susceptance values, the scatter between the exact values of zero and the educed values are reduced when compared to the upstream source eductions. This trends is not well understood and is under additional investigation. It is believed that the increased scatter in educed admittance at this Mach number when the upstream or downstream source is used is due to neglect of the mean flow gradients in the mean flow boundary layer. The effects of these flow gradients are not accounted for in our uniform flow impedance eduction model but the magnitude of these gradients do increase with increasing mean flow speeds (i.e., Mach number).

\section{Ceramic Tubular Liner}

Figure 7 shows the educed normalized resistance, $\theta$, and reactance, $\chi$, for the flow-off condition $\left(M_{0}=0.0\right)$, with the ceramic tubular liner installed in the GFIT. The impedances educed with the upstream, downstream, and dualsource are nearly identical. Figure 8 shows results for the ceramic tubular liner at Mach $0.3\left(M_{0}=0.3\right)$. Just as with the hard-wall insert, there is little discrepancy between the normalized resistance and reactance educed using either the upstream or downstream source. There are two exceptions to this statement, however. First, at the anti-resonant frequency (i.e., $f=2.0 \mathrm{kHz}$ ), there is some scatter in the educed impedance for the upstream and downstream source. This was expected because the liner produces little attenuation at the anti-resonant frequency. Second, there is scatter in the educed normalized resistance near $2.5 \mathrm{kHz}$ when the dual-source is used. This is due to the cut on of the next higher-order mode in the GFIT. This mode is not accounted for in the impedance eduction model as only the planewave mode is assumed cut on. Finally, a comparison of the current Mach 0.3 calculations with the Mach 0.0 results indicates the effective impedance (i.e., the curve containing the open circles) is closer to the Mach zero results. Thus, when the dual-source is used to educe an effective impedance, the ceramic liner appears slightly more linear than when the upstream or downstream source data is used. Finally, Fig. 9 shows results for the ceramic liner at the highest Mach number $\left(M_{0}=0.5\right)$. There is significant scatter in the normalized resistance and reactance educed when the upstream or downstream source is used. This discrepancy is believed to be due to the neglect of the mean flow gradients in the impedance eduction model, which become stronger at the higher Mach number.

\section{Low-Porosity Conventional liner}

In the next example, the Ingard-Myers impedance boundary condition is tested on the third liner (the conventional liner with the lowest porosity). Figure 10 shows the educed normalized resistance, $\theta$, and reactance, $\chi$, for the flow-off 
condition $\left(M_{0}=0.0\right)$, with the conventional liner installed in the GFIT. A few observations concerning the educed impedance are worth noting. First, as expected, the eduction procedure educes a low resistance for this flow-off condition. Second, the liner has been designed so that the anti-resonant frequency is outside the frequency range of interest. Third, the normalized resistance and reactance values educed using either the upstream or downstream source are nearly identical. Finally, no increased accuracy in educed impedance using the dual-source data (see the curve containing the open circles) is again observed.

Figure 11 shows results for the conventional liner at Mach $0.3\left(M_{0}=0.3\right)$. Just as with the hard-wall insert and the ceramic tubular liner, the impedances educed with the upstream and downstream sources are nearly identical. In fact, smaller discrepancies between the upstream and downstream source eductions are observed for the conventional liner than for the ceramic liner (see Fig. 8). As expected, there is a discrepancy at the frequency $(f=2.6 \mathrm{kHz})$ closest to the cut on of the next higher-order mode (see Fig. 11). Impedances educed at the highest Mach number $\left(M_{0}=0.5\right)$ are shown in Fig. 12 for the conventional liner. There is significant scatter in the normalized resistance and reactance educed when the upstream or downstream source is used. This discrepancy is believed due to the presence of gradients in the mean flow which are stronger at the higher Mach number.

\section{B. Comparison of the Ingard-Myers and Modified Ingard-Myers Boundary Conditions}

In this subsection, the modified Ingard-Myers impedance boundary condition is evaluated and compared to results of the Ingard-Myers boundary condition. Results in the previous subsection indicate that superior results can be obtained using an effective impedance that minimizes the dual-objective function formed from the dual-source data. Therefore, to limit the number of graphs, only the effective educed impedance is presented. As indicated by Eq. (5), there is no momentum transfer into a rigid wall when mean flow is presence $\left(M_{0} \neq 0.0\right)$. Additionally, there cannot be transfer of momentum into the liner (by the mean flow) when the flow is turned off. Therefore, the Ingard-Myers and modified Ingard-Myers boundary conditions are identical in these two environments (i.e., in a hard-wall duct and in a duct with a liner when the flow is turned off). Consequently, the evaluation in this subsection is confined primarily to those cases for which the flow is turned on (i.e., $M_{0}>0.0$ ) and the ceramic or conventional liner is installed.

\section{Ceramic Tubular Liner}

This liner is known to be very linear (i.e., its resistance and reactance spectra should be nearly independent of the flow Mach number). Therefore, the educed impedance in the presence of flow should track the no-flow values well for this liner. The normalized resistance and reactance spectra computed from both boundary conditions (Ingard-Myers and modified Ingard-Myers) are compared in Fig. 13 as a function of the mean flow Mach number. The largest discrepancy between the impedances educed in the no-flow and flow conditions is in the normalized resistance, especially near the anti-resonance frequency (i.e., $2.0 \mathrm{kHz}$ ). As mentioned earlier, discrepancies near anti-resonance are expected. The Mach 0.3 eductions for both boundary conditions are close to the zero flow results. Note that the Ingard-Myers Mach 0.3 results compare more favorably with the Mach 0.0 results, especially at the lower frequencies. The Mach 0.5 eductions are not in good agreement with the zero flow calculations. This is believed to be due to the neglect of the mean flow gradients at this higher Mach number.

\section{Low-Porosity Conventional Liner}

The modified Ingard-Myers impedance boundary condition and Ingard-Myers boundary condition are compared for the third liner (the conventional liner with the lowest porosity) in Fig. 14. The educed reactance is nearly independent of the flow Mach number when either boundary condition is used. Both boundary conditions predict a low resistance at Mach 0.0 (as expected) and this resistance increases with increasing mean flow Mach number (also expected). There is some unusual behavior in the low frequency end of the spectra at the higher Mach number when the modified IngardMyers boundary condition is used. Otherwise, similar results are achieved with each boundary condition provided an effective impedance is used. Further, the results presented in Fig. 6 of Ref. 12 seems to further corroborate this finding. Note that the impedance educed with the modified Ingard-Myers boundary condition presented in Fig. 6 of Ref 12 is simply an average of that educed using the Ingard-Myers boundary condition for the upstream and downstream source. This average impedance can be considered as the effective impedance for the Ingard-Myers boundary condition.

\section{The Momentum Transfer Coefficient}

Figure 15 shows the real and imaginary parts of the educed momentum transfer coefficient due to mean flow, $B$, for both the ceramic tubular and conventional liners. Results are presented using a dual-axis system, in which the real and 
imaginary parts of the momentum transfer coefficient due to mean flow, $B$, are presented on the left and right $y$-axes, respectively. For the ceramic tubular liner, the real part (Real $(B))$ of this coefficient hovers around a value of unity for both flow conditions (i.e., Mach 0.3 and Mach 0.5). The imaginary part is also quite small, except near the low and high frequency ends of the spectrum. For the conventional liner, the real part of the momentum transfer coefficient due to the mean flow hovers about a value of unity, and its imaginary part $(\operatorname{Imag}(B))$ is low at Mach 0.3.

\section{Concluding Remarks}

The suitability of the Ingard-Myers and modified Ingard-Myers boundary conditions for impedance eduction has been tested on four test samples, at three mean flow Mach numbers, and using upstream and downstream sound sources. Based upon the results of this study, the following conclusions can be drawn:

1. Discrepancies in educed impedance using upstream and downstream sound sources and the Ingard-Myers boundary condition do occur with data acquired in the LaRC Grazing Flow Impedance Tube. However, these discrepancies occur only at the highest Mach number (Mach 0.5).

2. It is believed that the discrepancies at Mach 0.5 observed in this study are due to the absence of mean flow gradients in the impedance eduction model (the effects of the mean flow gradients are strongest at the highest Mach number).

3. An effective Ingard-Myers impedance educed using dual-source (both upstream and downstream sources) data increases the accuracy of the impedance eduction model over that obtained with either an upstream or a downstream source.

4. In general, similar results are achieved with the Ingard-Myers and modified Ingard-Myers boundary conditions when dual-source (combination of upstream and downstream source data) is used to educe an effective impedance. Deviations between the two results are observed at the lowest frequencies considered in this study, especially as the Mach number is increased.

\section{Acknowledgement}

This work was funded by NASA's Subsonic Fixed Wing Project of the Fundamental Aeronautics Program.

\section{References}

${ }^{1}$ Kraft, R. E., "Theory and Measurements of Acoustic Wave Propagation in Multi-Segmented Rectangular Ducts," Ph.D. Dissertation, Department of Aerospace Engineering, University of Cincinnati, Cincinnati, Ohio, 1976.

${ }^{2}$ Ingard, K. U., "Influence of Fluid Motion Past a Plane Boundary on Sound Reflection, Absorption, and Transmission," Journal of the Acoustical Society of America, Vol. 31, No. 7, 1959, pp. 1035-1036.

${ }^{3}$ Myers, M. K., "On the Acoustic Boundary Condition in the Presence of Flow," Journal of Sound and Vibration, Vol. 71, No. 3, 1980, pp. $429-434$.

${ }^{4}$ Nayfeh, A. H., Kaiser, J. E., and Telionis, D. P., “The Acoustics of Aircraft Engine Ducts,” AIAA Journal, Vol. 11, No. 2, 1973 , pp. $150-153$.

${ }^{5}$ Dougherty, R. P., “A Wave-Splitting Technique for Nacelle Acoustic Propagation,” AIAA Paper 97-1652, 1997.

${ }^{6}$ Eversman, W. and Roy, I. D., "Ducted fan acoustic radiation including the effects of nonuniform mean flow and acoustic treatment," AIAA Paper 93-4424, 1993.

${ }^{7}$ Eversman, W. and Beckemeyer, R. J., "Transmision of Sound in Ducts with Thin Shear Layers - Convergence to the Uniform Flow Case," Journal of the Acoustical Society of America, Vol. 52, 1972, pp. 216-220.

${ }^{8}$ Brambley, E. J., "Fundamental Problem with the Model of Uniform Flow Over Acoustic Linings, "Journal of Sound and Vibration, Vol. 322, 2009, pp. 1026-1037.

${ }^{9}$ Marx, D., Auregan, Y., Bailliet, H., and Valiere, J., "PIV and LDV Evidence of Hydrodynamic Instability Over a Liner in a Duct with Flow," Journal of Sound and Vibration, Vol. 329, No. 18, 2010, pp. 3798-3812.

${ }^{10}$ Rienstra, S. W. and Darau, M., "Boundary-Layer Thickness Effects of the Hydrodynamic Instability Along an Impedance Wall," Journal of Fluid Mechanics, Vol. 671, 2011, pp. 559-573.

${ }^{11}$ Renou, Y. and Auregan Y., "Failure of the Ingard-Myers Boundary Condition for a Lined Duct: An Experimental Investigation," Journal of the Acoustical Society of America, Vol. 130, No. 1, 2011, pp. 52-60.

${ }^{12}$ Jing, X., Peng, S., and Sun, X., "A Straightforward Method for Wall Impedance Eduction in a Flow Duct," Journal of the Acoustical Society of America, Vol. 124, No. 1, 2008, pp.227-234.

${ }^{13}$ Watson, W. R., Jones, M. G., Tanner, S. E., and Parrott, T. L., "Validation of a Numerical Method for Extracting Liner Impedance," AIAA Journal, Vol. 34, No. 3, 1996, pp. 548-554. 
${ }^{14}$ Watson, W. R., Jones, M. G., Tanner, S. E., and Parrott, T. L., "A Finite Element Propagation Model for Extracting Normal Incidence Impedance in Nonprogressive Acoustic Wave Fields," Journal of Computational Physics, Vol. 125, Issue 1, 1996, pp. 177-186.

${ }^{15}$ Watson, W. R., Jones, M. G., and Parrott, T. L., "Validation of an Impedance Eduction Method in Flow," AIAA Journal, Vol. 37, No. 7, 1999, pp. $818-824$.

${ }^{16}$ Watson, W. R., Jones, M. G., and Parrott, T. L., "Comparison of a Convected Helmholtz and Euler Model for Impedance Eduction in Flow," AIAA Paper 2006-2643, 2006.

${ }^{17}$ Watson, W., Jones, M., and Parrott, T., "Investigation of an Anomaly Observed in Impedance Eduction Techniques," AIAA Paper 2008-3013, 2008.

${ }^{18}$ Elnady,T., Musharraf, M., Boden, H., and Elhadidi, B., "Validation of an Inverse Analytical Technique to Educe Liner Impedance with Grazing Flow," AIAA Paper 2006-2639, 2006.

${ }^{19}$ Jones, M. G., Watson, W. R., and Nark, D. M., "Effects of Flow Profile on Educed Acoustic Liner Impedance," AIAA Paper 2010-3763, 2010.

${ }^{20}$ Stewart, G. W., III, “A Modification of Davidon's Minimization Method to Accept Difference Approximations of Derivatives," Journal of ACM, Vol. 14, No. 1, 1967, pp. 72-83.

${ }^{21}$ Goldberg, D., Genetic Algorithms in Search, Optimization and Machine Learning, Addison-Wesley, New York, NY, 1989 , pp. $75-147$.

\section{Figures}

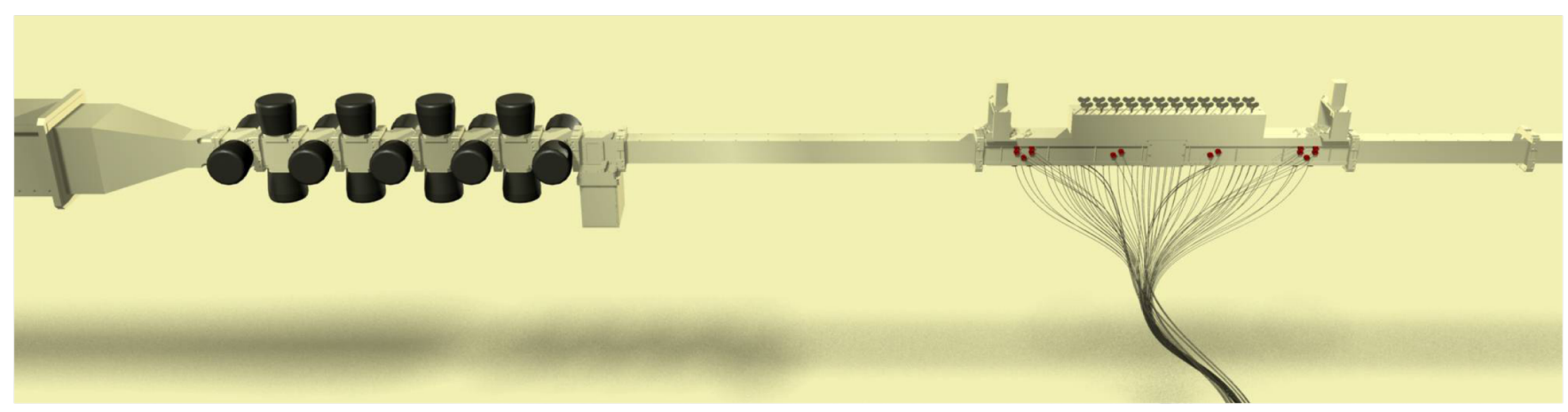

Figure 1. Sketch of the LaRC grazing flow impedance tube (GFIT) and instrumentation.

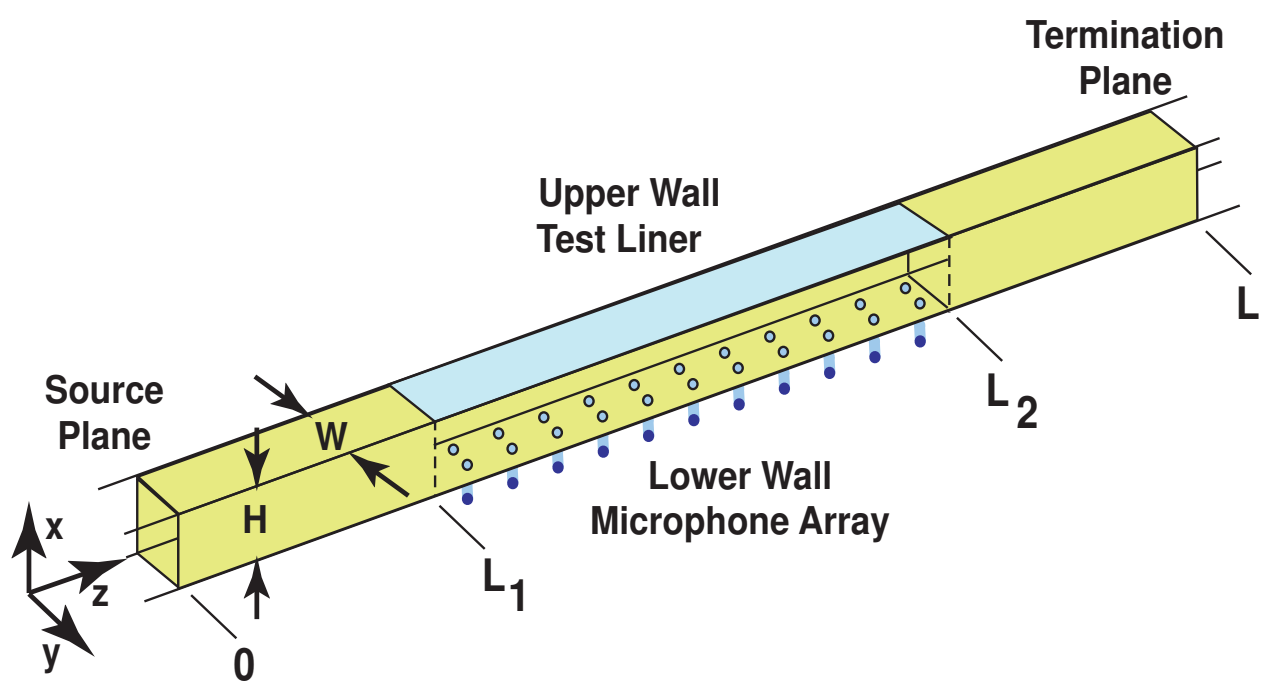

Figure 2. Schematic of the LaRC grazing flow impedance tube (GFIT) flow duct. 


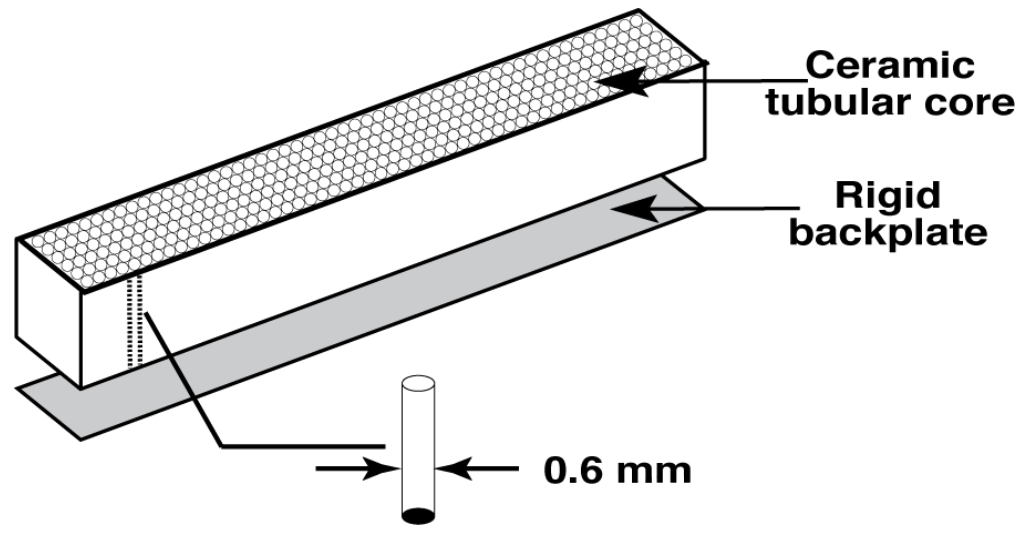

(a) Ceramic tubular liner

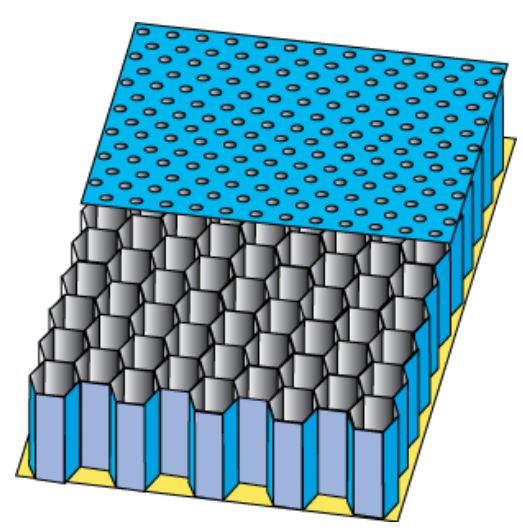

(b) Conventional liner

Figure 3. Schematic of test liners.

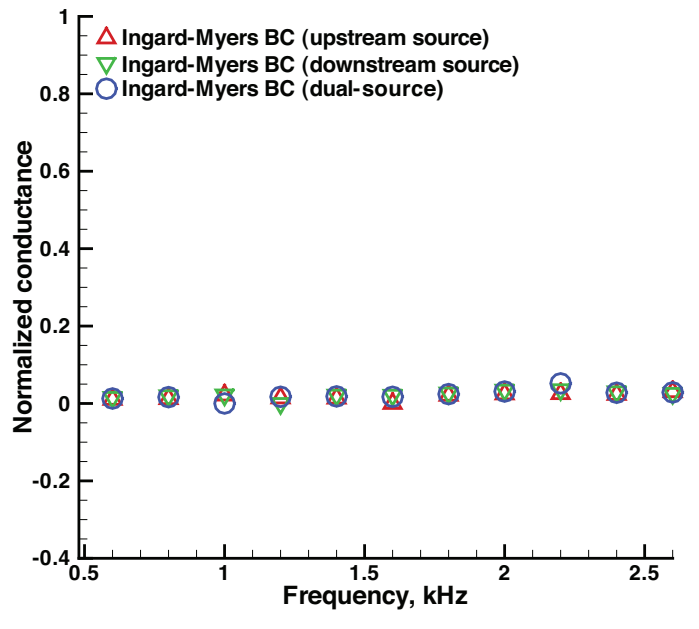

(a) Normalized conductance

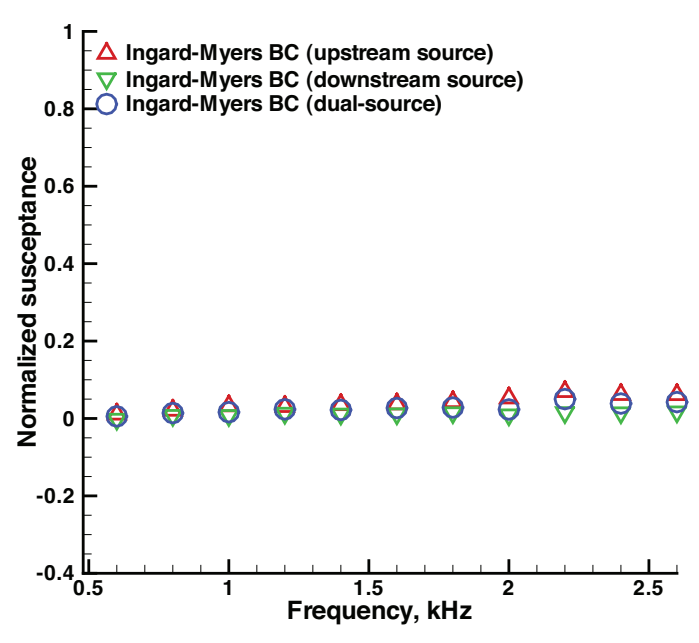

(b) Normalized susceptance

Figure 4. Educed normalized conductance and susceptance of hard-wall insert at Mach 0.0 . 


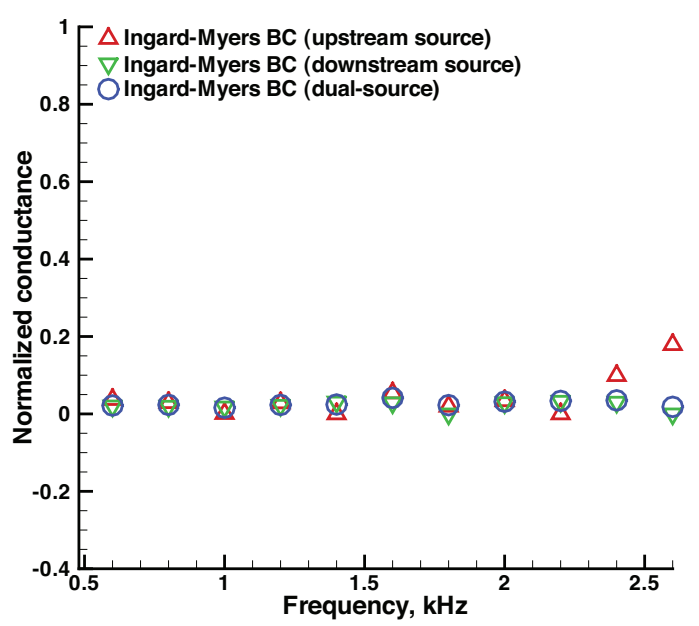

(a) Normalized conductance

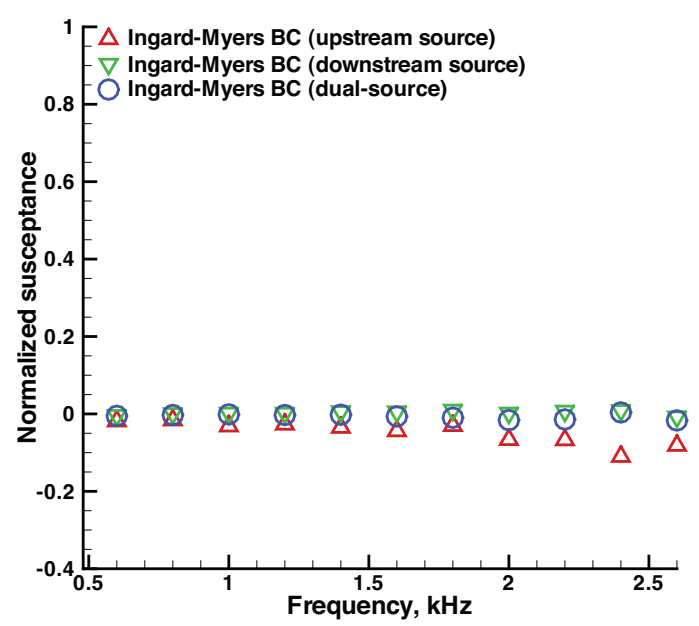

(b) Normalized susceptance

Figure 5. Educed normalized conductance and susceptance of hard-wall insert at Mach 0.3.

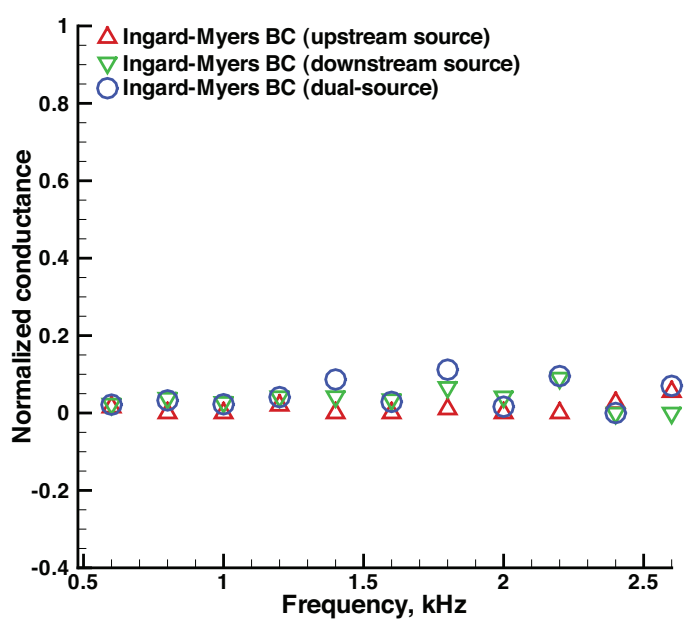

(a) Normalized conductance

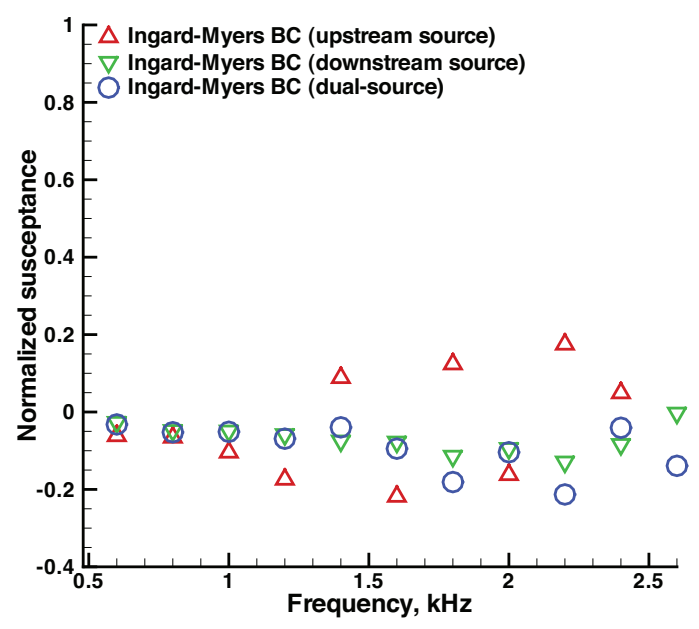

(b) Normalized susceptance

Figure 6. Educed normalized conductance and susceptance of hard-wall insert at Mach 0.5 . 


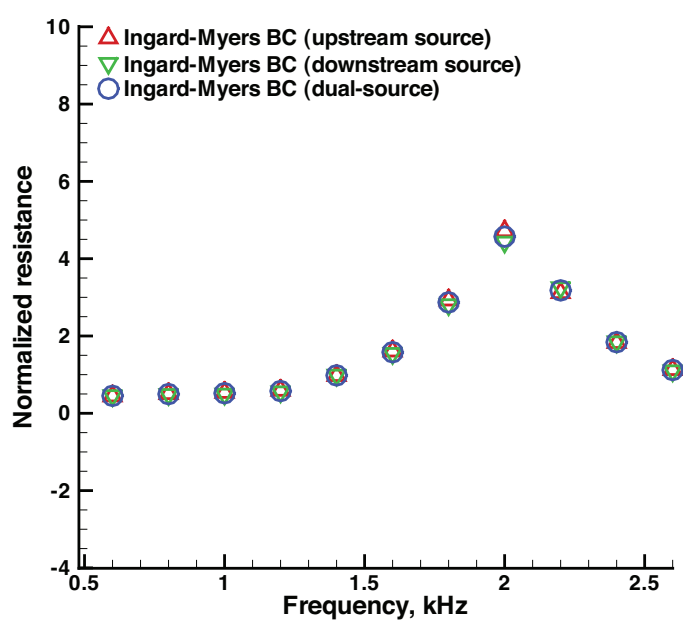

(a) Normalized resistance

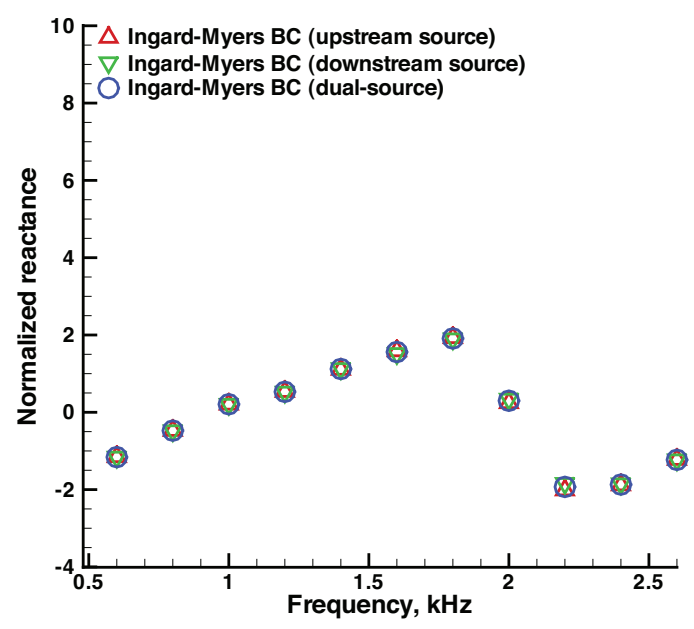

(b) Normalized reactance

Figure 7. Educed normalized resistance and reactance of ceramic tubular liner at Mach 0.0.

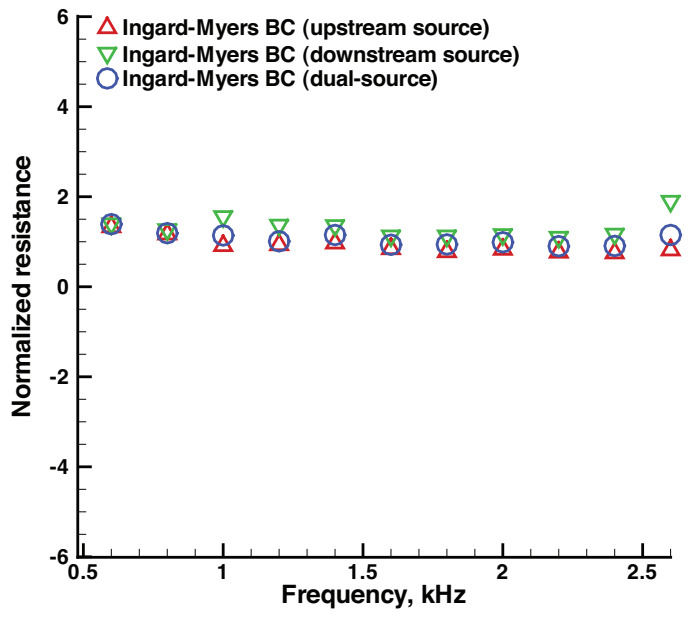

(a) Normalized resistance

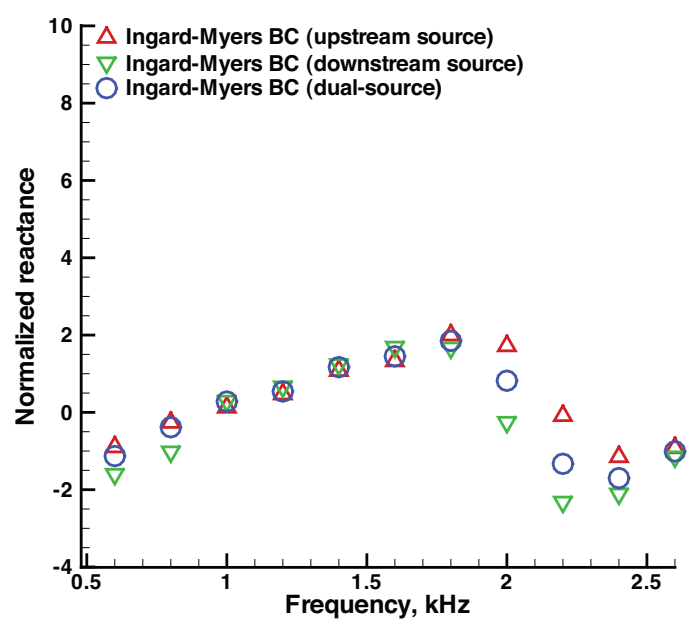

(b) Normalized reactance

Figure 8. Educed normalized resistance and reactance of ceramic tubular liner at Mach 0.3. 


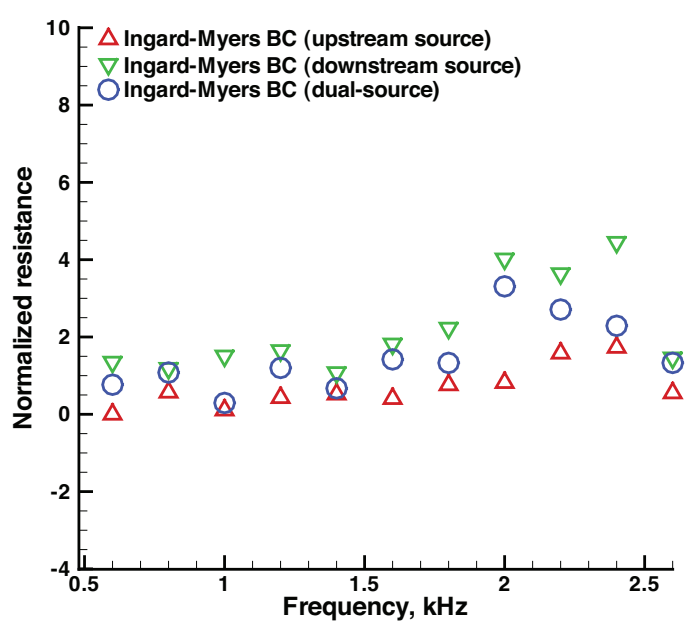

(a) Normalized resistance

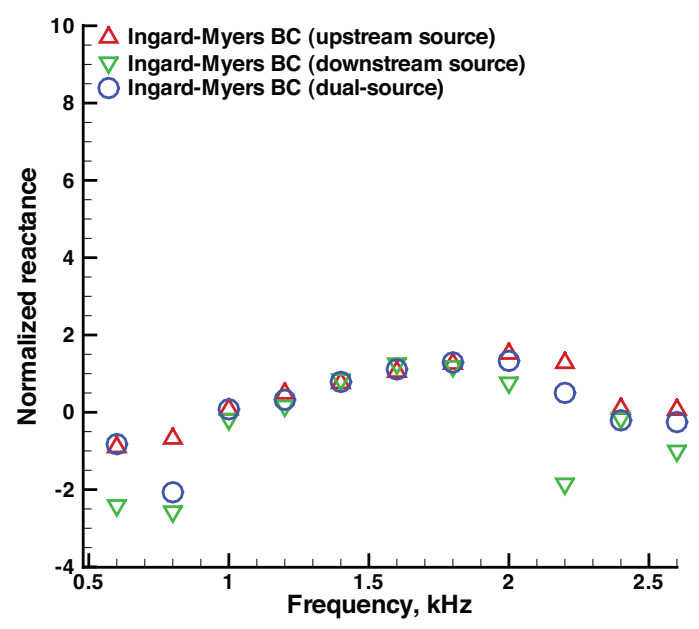

(b) Normalized reactance

Figure 9. Educed normalized resistance and reactance of ceramic tubular liner at Mach 0.5 .

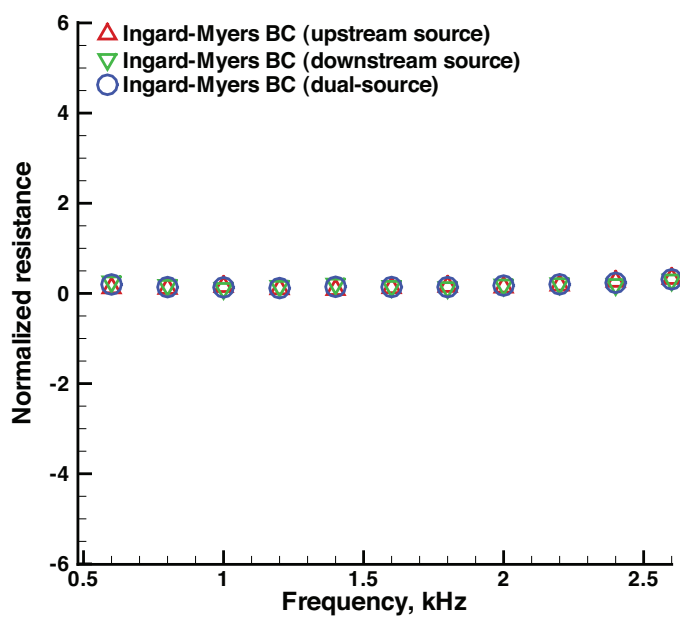

(a) Normalized resistance

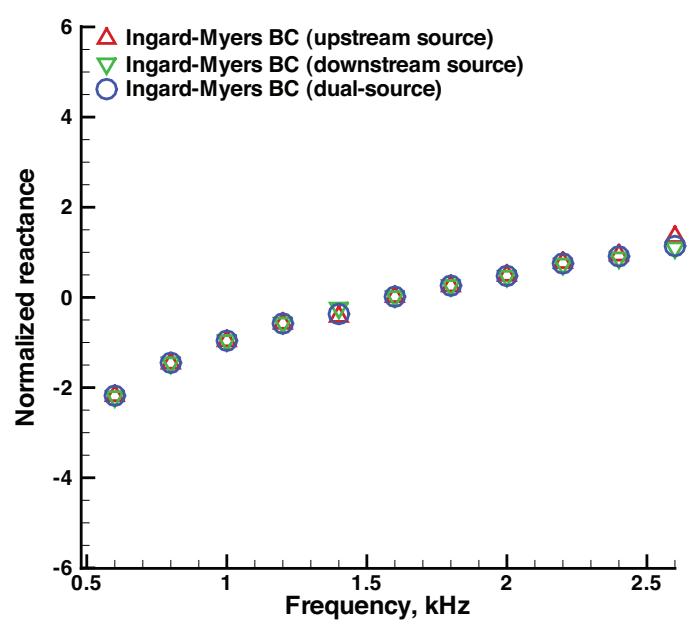

(b) Normalized reactance

Figure 10. Educed normalized resistance and reactance of conventional liner at Mach 0.0 . 


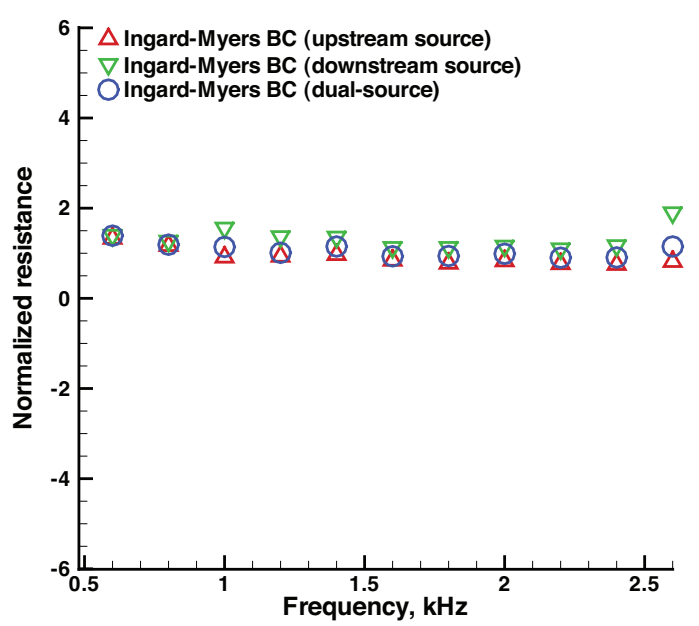

(a) Normalized resistance

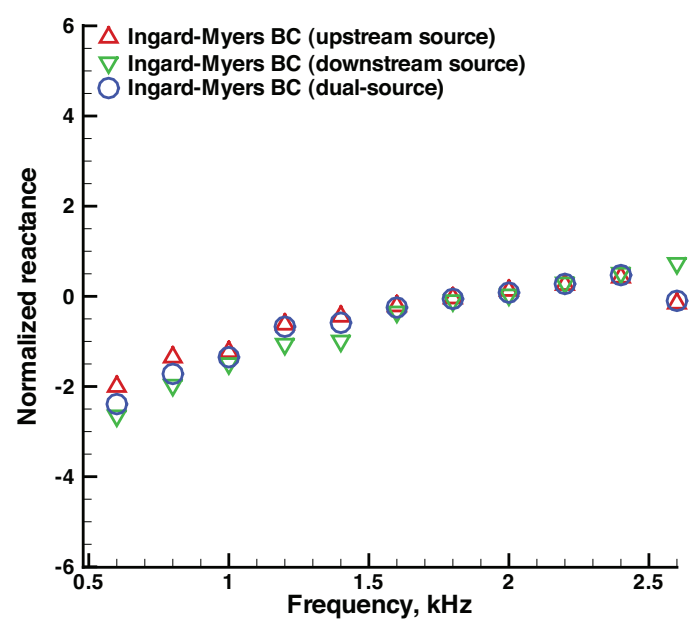

(b) Normalized reactance

Figure 11. Educed normalized resistance and reactance of convential liner at Mach 0.3.

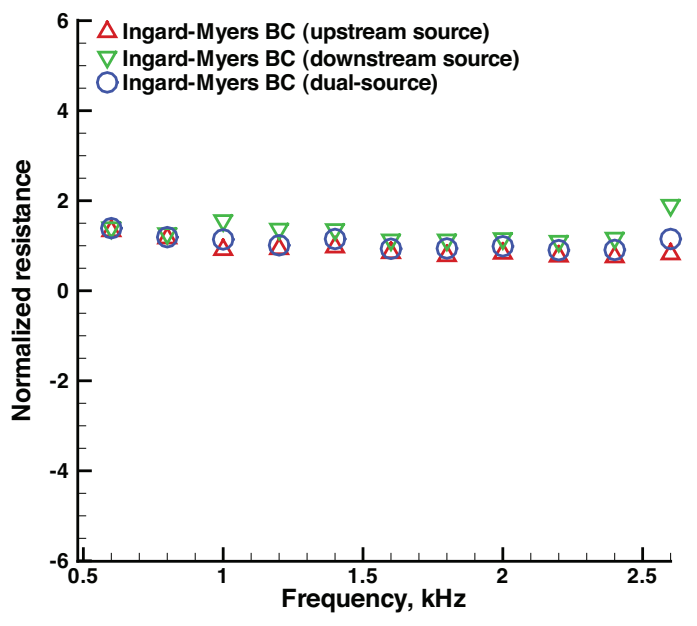

(a) Normalized resistance

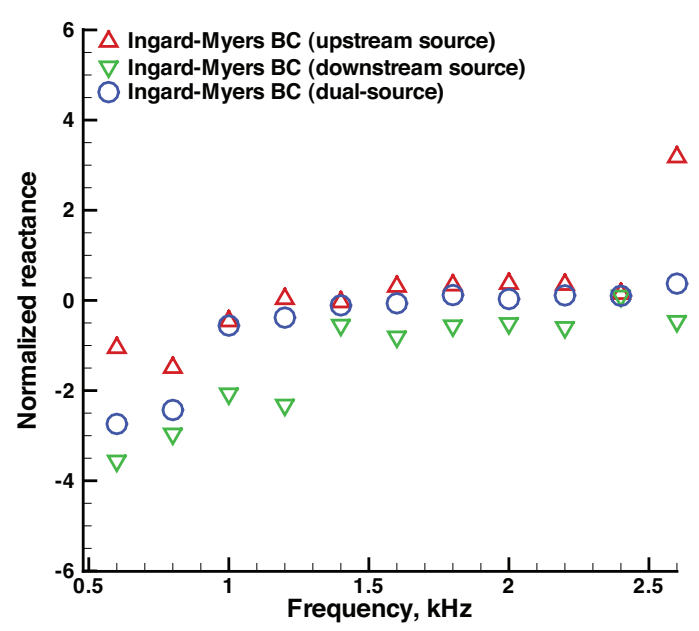

(b) Normalized reactance

Figure 12. Educed normalized resistance and reactance of conventional liner at Mach 0.5. 


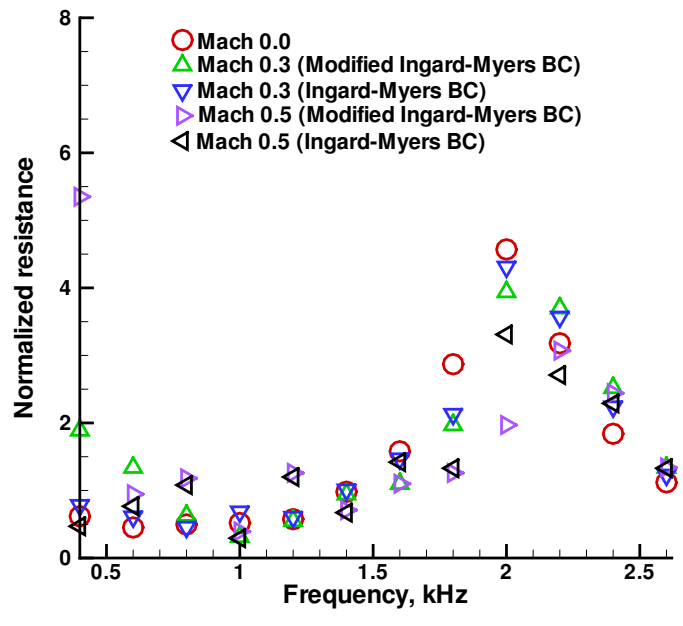

(a) Normalized resistance

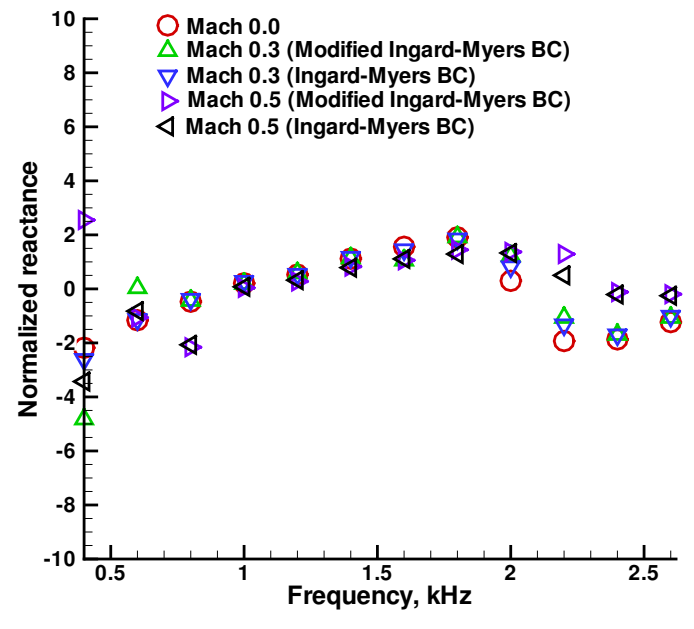

(b) Normalized reactance

Figure 13. Educed normalized resistance and reactance for the ceramic tubular liner using both upstream and downstream source data and both boundary conditions.

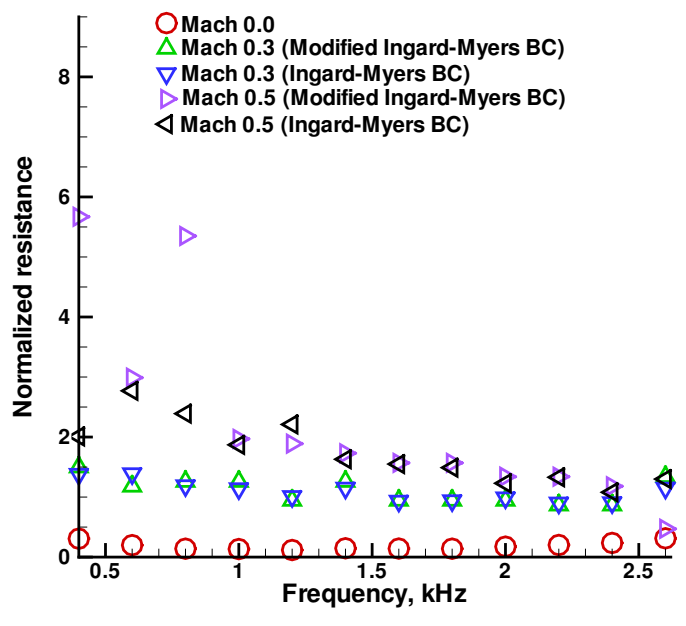

(a) Normalized resistance

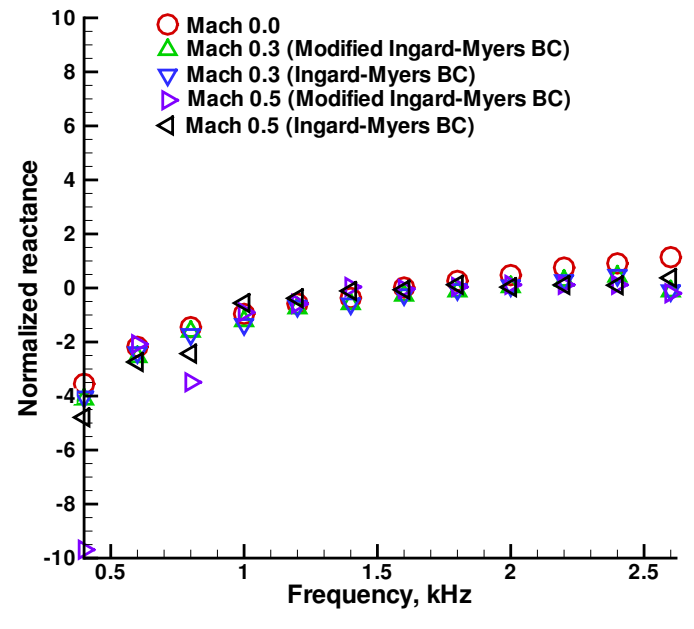

(b) Normalized reactance

Figure 14. Educed normalized resistance and reactance for the conventional liner using both upstream and downstream source data and both boundary conditions. 


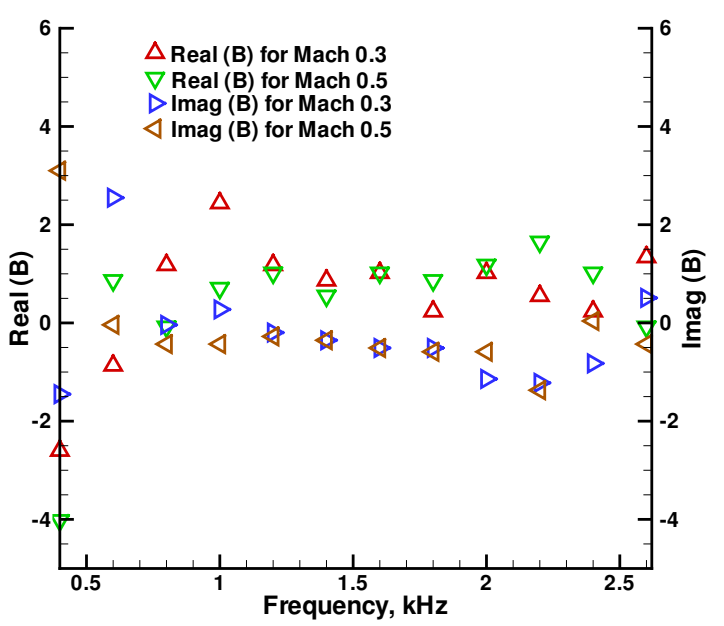

(a) Ceramic tubular liner

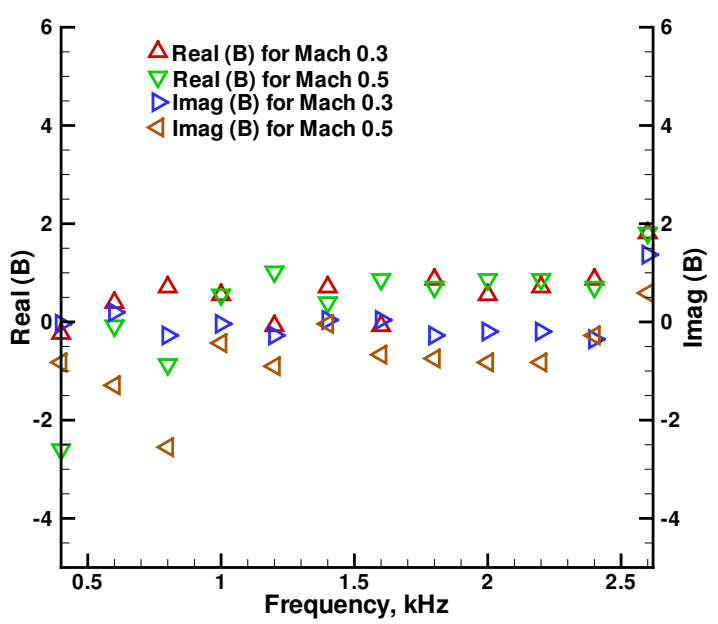

(b) Conventional liner

Figure 15. Educed momentum transfer coefficient due to mean flow, $B$, for ceramic tubular and conventional liner. 